\title{
African star joins the radio astronomy firmament
}

\author{
A powerful new radio telescope will improve our understanding of galaxy formation and evolution, and other key \\ questions in astrophysics, says Fernando Camilo, on behalf of the MeerKAT team.
}

A fter a decade in the works, South Africa's MeerKAT telescope (Fig. 1), a precursor to the Square Kilometre Array (SKA) mid-frequency telescope, is beginning science operations. MeerKAT is a radio interferometer located in the semiarid and sparsely populated Karoo region of the Northern Cape. The array consists of 64 antennas $13.5 \mathrm{~m}$ in diameter located on baselines of up to $8 \mathrm{~km}$.

The distribution of the antennas, with three quarters of them located within a 1-km-diameter core, makes MeerKAT particularly suited to a variety of pulsar and neutral hydrogen studies. Several of the selected large survey projects (LSPs), which will use two thirds of the available observing time within five years, will address key questions related to galaxy formation and evolution. For instance, the unique combination of column density sensitivity and angular resolution will make MeerKAT a powerful probe for studying accretion onto galaxies in the nearby Universe. Projects will investigate the range of conditions from star-forming disks to low-density gas in dark matter haloes in isolated galaxies, and will examine how galaxies interact within rich clusters, while seeking to detect the cosmic web. Further afield, the $21-\mathrm{cm}$ line of neutral hydrogen will be used to investigate the properties and evolution of galaxies across two thirds of cosmic time.

Although optimized for particular applications, MeerKAT is an extremely sensitive general-purpose radio telescope. The dishes are of a highly efficient offset Gregorian design, with up to four receiver systems positioned on a turret near the subreflector, without compromising the clean optical path. Three cryogenic receivers are currently being installed: $\mathrm{L}$ band (covering 900-1,670 MHz), UHF (580-1,015 MHz) and $\mathrm{S}$ band (1.75-3.5 GHz; provided by the Max Planck Institute for Radio Astronomy). The measured sensitivity of one $13.5-\mathrm{m}$ MeerKAT antenna at L band is comparable to that of a 25-m Jansky Very Large Array antenna, with a much larger field of view.

The radio-frequency voltages for each of two linear polarizations are digitized in a shielded package just $1 \mathrm{~m}$ behind the receivers. The samples are then transmitted via buried optical fibre to the Karoo Array Processor Building (KAPB), located $\sim 10 \mathrm{~km}$ away. There, the MeerKAT FX/B

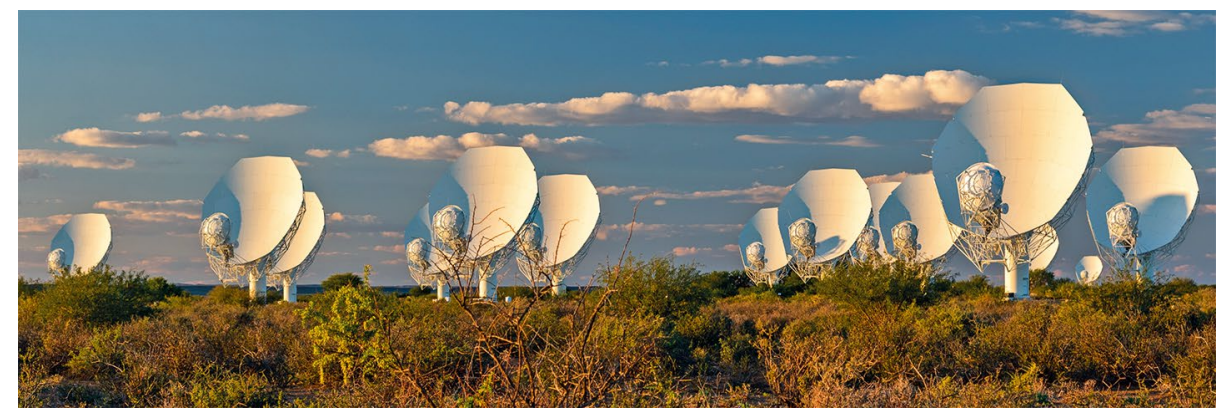

Fig. 1 | Part of the 64-dish MeerKAT array. Credit: South African Radio Astronomy Observatory

correlator/beamformer processes the 2 Tbps of data arriving from 64 dishes in real time. The correlator is based on the CASPER (Collaboration for Astronomy Signal Processing and Electronics Research) architecture, using Ethernet switches to handle data transfer between processing nodes. For MeerKAT, the processing nodes consist of SKARAB (SKA Reconfigurable Application Board) boards populated with field-programmable gate arrays, developed at SKA South Africa.

The flexibility of the MeerKAT architecture enables the deployment of multiple guest instruments that can subscribe to telescope data streams and simultaneously process them in a bespoke fashion tuned to each specific scientific requirement. In this way, for instance, while one LSP investigates the evolution of the cosmological star-formation rate density (through a continuum survey of selected fields), another team searches for fast radio bursts lasting a few milliseconds (which may be precisely localized because the back-end forms hundreds of beams tiling the large primary telescope beam), while yet another group searches for artificial signals from extraterrestrial sources.

The MeerKAT science data processor receives up to $300 \mathrm{Gbps}$ from the correlator, which it averages in real time after applying appropriate flagging and calibration solutions. The resulting $\sim 10$ Gbps streams are archived, and further processed through imaging pipelines to produce 'spectral cubes': maps of sky brightness as a function of frequency. The scale of the computational and storage challenge is significant, with $10^{15}$ floating-point operations per second of computing power deployed at the KAPB, and $40 \mathrm{~PB}$ of storage at the Centre for High Performance Computing in Cape Town, where the permanent MeerKAT archive resides. Efficiency in both power and cost has been the dominant driving factor behind these solutions. For instance, careful specification, in-house integration and co-design with the software stack have yielded a massive-scale, high-performance storage cluster for a fifth of the cost of comparable commercial options.

MeerKAT is a South African-funded project, largely designed and built in South Africa. It is the result of an integrated plan that included careful consideration of multiple design options, development of technology demonstrators, training and investment in people through a human capital development programme and cooperation with industrial partners, and support from a wide variety of national stakeholders. First light occurred in 2016 using 16 antennas; the first science results were published in April 2018 (F. Camilo et al., Astrophys. J. 856, 180; 2018); and data collection has begun for the first LSPs using 64 antennas. The first call for open time proposals, for one-third of the available observing time, is expected in late 2018. Eventually, MeerKAT will be integrated into the international SKA Phase 1 mid-frequency array.

Fernando Camilo

South African Radio Astronomy Observatory

(SARAO), Cape Town, South Africa.

e-mail:fernando@ska.ac.za

Published online: 3 July 2018

https://doi.org/10.1038/s41550-018-0516-y 